\title{
Controle postural em crianças com paralisia cerebral e desenvolvimento típico
}

\author{
Postural control in children with cerebral palsy and typically developing children \\ Leonardo George Victorio Vitor', Rubens Alexandre da Silva Junior ${ }^{2}$, Lilian \\ Gerdi Kittel Ries', Dirce Shizuko Fujisawa
}

\section{RESUMO}

Objetivo. Verificar o comprometimento do controle postural em crianças com paralisia cerebral. Método. 64 crianças foram avaliadas e divididas em quatro grupos G1: cinco e seis; G2: sete e oito; G3: nove e dez anos, com desenvolvimento típico, e; G4: cinco a 12 anos, com paralisia cerebral. O controle postural foi avaliado por meio da Escala de Equilíbrio Pediátrica, Teste de Alcance e Plataforma de Força. Resultados. O déficit no controle postural foi identificado no G4, na escala de equilíbrio pediátrica. O G4 obteve 52 pontos, quando comparadas as crianças do G2 $(55,5)$ e G3 (56). No teste de alcance anterior houve diferença para o G4 $(19 \mathrm{~cm})$ em relação ao G2 $(25,5 \mathrm{~cm})$. Sobre a plataforma de força, na postura bipodal, a área do centro de pressão do G2 $\left(1,62 \mathrm{~cm}^{2}\right)$ e G3 $\left(1,36 \mathrm{~cm}^{2}\right)$ foi menor que do G4 $\left(4,24 \mathrm{~cm}^{2}\right)$. Conclusáo. A criança com paralisia cerebral tem prejuízo no controle postural, na realizaçáo de atividades funcionais, mobilidade e área do centro de pressão, e seu desempenho aproximou-se das crianças com desenvolvimento típico na faixa etária entre cinco e seis anos.

Unitermos. Criança, Paralisia Cerebral, Equilíbrio Postural

Citaçáo. Vitor LGV, Silva-Junior RA, Ries LGK, Fujisawa DS. Controle postural em crianças com paralisia cerebral e desenvolvimento típico.

\begin{abstract}
Objective. To assess the impairment of postural control in children with cerebral palsy. Method. 64 children were evaluated and divided into four groups G1: five to six; G2: seven to eight; G3: nine to ten years, with typical development and; G4: five to 12 years, with cerebral palsy. Postural control was evaluated by the Pediatric Balance Scale, Reach Test and Force Platform. Results. The deficit in postural control was identified in the G4, the pediatric balance scale G4 got 52 points, compared the children of the G2 (55.5) and G3 (56) resulted difference; in the above range test G4 $(19 \mathrm{~cm})$ and G2 $(25.5 \mathrm{~cm})$ were different; on the force platform, the bipedal posture, the area of G2 center of pressure $\left(1.62 \mathrm{~cm}^{2}\right)$ and G3 $\left(1.36 \mathrm{~cm}^{2}\right)$ were lower than the G4 $\left(4.24 \mathrm{~cm}^{2}\right)$. Conclusion. Children with cerebral palsy have impairment in postural control during functional activities, mobility and pressure center area, and its performance approached the typically developing children aged between five and six years.
\end{abstract}

Keywords. Children, Cerebral Palsy, Postural Balance

Citation. Vitor LGV, Silva-Junior RA, Ries LGK, Fujisawa DS. Postural control in children with cerebral palsy and typically developing children
Trabalho realizado na Universidade Estadual de Londrina, Londrina-PR, Brasil.
Endereço para correspondência: Dirce Shizuko Fujisawa Rua Borba Gato, 70 ap 204 CEP 86010-630, Londrina-PR, Brasil email:dirce_fujisawa@uel.br

\footnotetext{
1.Fisioterapeuta, Doutorando, Universidade Estadual de Londrina, Londrina-PR, Brasil.

2.Fisioterapeuta, Pós Doutor, Universidade Norte do Paraná, Londrina-PR, Brasil.

3.Fisioterapeuta, Doutora, Universidade do Estado de Santa Catarina, Florianópolis-SC, Brasil.

4.Fisioterapeuta, Doutora, Universidade Estadual de Londrina, Londrina-PR, Brasil.
} 


\section{INTRODUÇÃO}

As crianças passam por mudanças constantes até chegarem à fase adulta, cada etapa do desenvolvimento motor corresponde ao surgimento de novas habilidades e a melhoria do seu desempenho. Tal fato pode ser exemplificado pela aquisição da posição bípede, alcançada por volta do primeiro ano de vida, tarefa considerada difícil. No entanto, nem toda criança se desenvolve normalmente, algumas apresentam interferências ${ }^{1}$. Essas interferências podem ser resultantes de lesões durante o processo de maturação, como por exemplo, a paralisia cerebral (PC), que desencadeia anormalidades nos sistemas, como alteraçóes do tônus muscular, precariedade de sinergismo e diminuição de amplitude de movimentos, entre outras ${ }^{2}$.

A PC é uma lesão não progressiva do Sistema Nervoso Central (SNC), com prevalência de dois para cada 1000 nascidos vivos em países industrializados, podendo ocorrer entre o período fetal e o segundo ano de vida, geralmente, acompanhada de distúrbios associados, que comprometem a aquisição de habilidades e limitam o desenvolvimento motor ${ }^{3-5}$. Dentre as varias consequências da PC sobre o desenvolvimento e aprendizagem motora, está o comprometimento das reações de equilíbrio, em função das manifestaçôes clinicas ${ }^{6}$.

O equilíbrio é o momento de estabilidade, em que as quedas não ocorrem. As respostas motoras, após receber informaçôes provenientes dos sistemas vestibular, proprioceptivo e visual, são formas de o indivíduo realizar o controle postural, alinhando o equilíbrio que foi colocado em instabilidade 7 . Quanto mais velhas as crianças, melhor será o seu controle postural, devido à maturação dos sistemas, conforme a progressão das etapas do desenvolvimento $^{1,7}$.

O equilíbrio tem sido demonstrado como habilidade essencial durante a realização das atividades de vida diária de crianças, tanto em desenvolvimento típico (DT), quanto com déficits. Assim, a avaliação clínica e objetiva do equilíbrio é fundamental, sendo necessários instrumentos, tais como escalas e testes, que possibilitem o diagnóstico, identifiquem as causas das perturbaçóes e avaliem a eficácia do tratamento proposto ${ }^{8}$. Entretanto, os testes funcionais, em sua maioria, identificam a existência do déficit de equilíbrio, mas podem não determinar a sua magnitude. A avaliação ideal deve fornecer dados qualitativos e quantitativos por medidas computadorizadas, como por exemplo, a plataforma de força $(\mathrm{PF})^{9}$.

Os estudos de equilíbrio em crianças com PC apresentam, na maioria, amostras que variam entre seis a 20 crianças, ainda, utilizando somente instrumentais clínicos ou a plataforma de força (PF). Portanto, em função da importância do equilíbrio nas atividades do cotidiano, julga-se necessário observar o comportamento dessa variável em crianças com PC e desenvolvimento motor típico (DT), utilizando escalas funcionais e subjetivas (Escala de Equilíbrio Pediátrica e Teste de Alcance), que são de fácil acesso, quanto aos parâmetros objetivos, como a PF, mais precisa. Os objetivos do estudo proposto foram determinar se crianças com PC apresentam déficits no controle postural quando comparadas as crianças com DT, e, também estabelecer a maturação do equilíbrio, conforme o avançar da idade dos grupos em DT.

\section{MÉTODO}

\section{Amostra}

O estudo realizado foi do tipo transversal, tendo sido a amostra de conveniência. Foram avaliadas 64 crianças de ambos os sexos, na faixa etária entre cinco e 12 anos, provenientes das escolas da Rede Municipal de Ensino de Londrina-PR e dos serviços de Fisioterapia. Os critérios de exclusão foram crianças com diagnostico de doenças aguda ou crônicas, fraturas ósseas recentes ou deformidades e contraturas, incapazes de deambular e permanecer na posiçáo de pé independentemente da utilização de órteses, com déficit cognitivo ou de atenção e submetidas a cirurgias ortopédicas ou toxina botulínica nos últimos seis meses.

O projeto de pesquisa e o termo de consentimento livre e esclarecido foram aprovados pelo Comitê de Ética da Universidade Estadual de Londrina/Hospital Universitário (Parecer 020/2010). A Secretaria Municipal de Educação de Londrina-PR autorizou o desenvolvimento do projeto de pesquisa nas escolas do município. Todos os pais ou responsáveis autorizaram a realização da avaliaçáo e assinaram o termo de consentimento livre e esclarecido. 


\section{Procedimento}

Os participantes foram divididos em quatro grupos, conforme a faixa etária e a presença de disfunção motora: G1: cinco e seis anos; G2: sete e oito anos; G3: nove e dez anos, com desenvolvimento típico e; G4: cinco e 12 anos, com diagnostico de PC. Cada grupo continha 16 participantes totalizando 64 . As crianças com PC foram agrupadas independente da classificação topográfica e grau de comprometimento.

Os dados de identificação foram coletados por meio de questionários, aplicados previamente, para verificar a presença de critérios de exclusão e respondidos pelos pais. O exame físico constituiu-se de mensuração do peso em quilogramas, G1: mediana 19,4 (18,2;21,3); G2: 20,4 (22,0;28,3); G3: 36,1 (30,7;43,3); G4: 26,8 (21,5;31,0); e altura em metros, G1: mediana 1,1 $(1,1 ; 1,2)$; G2 1,3 $(1,2 ; 1,3)$; G3: $1,4(1,4 ; 1,5)$; G4: 1,3 $(1,2 ; 1,4)$.

Para classificar o grau de comprometimento motor do G4 foi utilizado a Escala de Função Motora Grossa (GMFCS), já o equilíbrio e o controle postural foram avaliados pela Escala de Equilíbrio Pediátrica (EEP), Teste de Alcance (TA) e Plataforma de Força (PF).

\section{Gross Motor Function Classification System}

Versão brasileira da Gross Motor Function Classification System, adaptada transculturalmente para o português-brasileiro3, com o proposito de estabelecer o grau de comprometimento motor da criança com PC. A caracterização do comprometimento do G4 está descrito na Tabela 1.

\section{Escala de Equilíbrio Pediátrica}

Versão brasileira da escala de Equilíbrio Pediátrica, adaptada transculturalmente e validada ${ }^{10}$. A EEP é de fácil aplicação, constituída de 14 itens, que podem ser concluídas em 20 minutos, tem como propósito testar o equilíbrio na realização das atividades de vida diária, ou seja, trata-se de avaliação funcional. Os itens evoluem de posiçôes estáveis, como por exemplo, a posição sentada, para posição mais instáveis, como a posiçẫo vertical. As pontuaçôes variam de zero a quatro, a atribuição de maior valor em cada item corresponde ao melhor desempenho, sendo o escore máximo 56. Nesse sentido, quanto maior o escore final, melhor terá sido o desempenho.

\section{Teste de Alcance}

O Teste de Alcance (TA) ${ }^{11}$ avalia o equilíbrio dinâmico, que é definido como a distância máxima possível de atingir, além do comprimento do membro superior, mantendo-se a base de apoio fixa. A fita métrica foi fixada na parede, na altura do processo acrômial do membro superior dominante da criança, com ombro em $90^{\circ}$ de flexão (anterior) e abdução (direita e esquerda) ${ }^{11,12}$. A criança foi instruída a "chegar tão longe quanto possível" e manter-se por três segundos, sem tocar ou apoiar e permanecer com os pés em contato total com o chão, tendo sido incentivada verbalmente durante a execução. A diferença entre a posição inicial e a final do terceiro metacarpiano foi mensurada e registrada, o que corresponde a distância percorrida no alcance, tendo sido considerada o maior valor entre as duas tentativas. O TA tem como

Tabela 1. Distribuição do G4 quanto a forma de PC, topografia.

\begin{tabular}{lccccccc}
\hline $\mathbf{N}$ & FORMA DE PC & TOPOGRAFIA & GMFCS & N & FORMA DE PC & TOPOGRAFIA & GMFCS \\
\hline $\mathbf{1}$ & ESPÁSTICO & HE & II & 9 & ESPÁSTICO & DI & III \\
$\mathbf{2}$ & ESPÁSTICO & HE & III & 10 & ESPÁSTICO & HE & III \\
$\mathbf{3}$ & ESPÁSTICO & HE & II & 11 & ESPÁSTICO & HD & II \\
$\mathbf{4}$ & ESPÁSTICO & HD & II & 12 & ESPÁSTICO & DI & II \\
$\mathbf{5}$ & ESPÁSTICO & HE & III & 13 & ESPÁSTICO & DI & II \\
$\mathbf{6}$ & ESPÁSTICO & DI & I & 14 & ESPÁSTICO & DI & III \\
$\mathbf{7}$ & ESPÁSTICO & DI & III & 15 & ESPÁSTICO & DI & III \\
$\mathbf{8}$ & ESPÁSTICO & HD & II & 16 & ESPÁSTICO & HD & I \\
\hline
\end{tabular}

HE (hemiplégico esquerdo); HD (hemiplégico direito); DI (diplégico) e grau de comprometimento motor GMFCS, I e II (leve) e III (moderado). 
objetivo avaliar o equilíbrio e a mobilidade da crianças, visto que alcançar o máximo possível desestabiliza o centro de massa e a coloca em situação de instabilidade anterior (TA_ANT); lateral direito (TA_D) e lateral esquerdo (TA_E).

\section{Plataforma de Força}

O controle postural foi quantificado conforme a distribuição de força vertical em quatro pontos, com a finalidade de obter dados objetivos e sensíveis. A PF utilizada foi a BIOMEC400, desenvolvida pela EMG System do Brasil, Ltda. (SP), com dois sensores para registros dos contatos dos pés no solo e canais configurados para força, com filtros em banda de frequência entre zero e $35 \mathrm{~Hz}$. Os principais parâmetros extraídos e analisados pela estabilografia são: área do Centro de Pressão (COP) e velocidades médias de oscilaçóes posturais, ambas nos planos anteroposterior e médio lateral e tempo de permanência na posição durante os testes. Os sinais da plataforma foram processados e tratados pelo próprio sistema de análise estabilográfica BIOMEC400. Os parâmetros são válidos e confiáveis para avaliação do equilíbrio ${ }^{13}$.

O protocolo de avaliação foi padronizado quanto ao tempo de permanência (15 segundos para cada postura) e nas posiçôes bipodal olhos abertos (BOA); unipodal direito (UNID) e esquerdo (UNIE). O numero de tentativas foi estipulado em dois, para a análise foi utilizada a média das duas tentativas. As avaliaçóes foram realizadas em ambiente tranquilo e os participantes ficaram com os pés descalços, olhar voltado a um ponto de referência na altura dos olhos, os braços eram mantidos ao longo do corpo, soltos e relaxados ${ }^{14}$ e com distância frontal de $2,5 \mathrm{~m}$.
Todas as avaliações foram realizadas por equipe treinada para o seguimento correto dos procedimentos padronizados para a coleta de dados, e também orientados quanto ao posicionamento próximo para evitar quedas das crianças, porem sem interferir na sua execução.

\section{Análise estatística}

Para comparaçáo do controle postural intergrupo foi realizado o teste de Kruskal-Wallis, conforme a não normalidade dos dados e, posteriormente, o teste de Dunn para localizar as diferenças intragrupos em cada variável estudada. A significância estatística foi estabelecida em $\mathrm{p}<0,05$. Os resultados são apresentados em medianas e quartis $(25 \%$ e $75 \%)$.

\section{RESULTADOS}

As crianças do G4 apresentam déficits de controle postural, quando comparadas a dos demais grupos nos testes funcionais. No G1, G2 e G3, as crianças mostram uma evolução com o avançar da idade, melhorando o desempenho de controle postural quando submetidas a testes funcionais (Tabela 2).

Nos testes posturográficos, nos momentos $\mathrm{BOA}$ e UNID, não foram observadas diferenças entre crianças com G4 e G1, G2 e G3, mas identificou-se a proximidade das medianas do grupo G4 e G1, que é o mais imaturo (Figuras 1 e 2). No teste UNIE as crianças de G4 não apresentaram pior controle postural em relação aos outros grupos. Também se confirmou que o avançar da idade influência positivamente no controle postural de crianças de G1, G2 e G3, quando submetidas a testes posturográficos (Figura 3).

Tabela 2. Resultados dos testes Funcionais. (EEP pontuação TA_ANT_D_E em cm alcançados) em medianas e percentis (25\% e $75 \%)$, valor de p Kruskal - Wallis.

\begin{tabular}{lcccccccc}
\hline & EEP & $\mathbf{2 5 ; 7 5 \%}$ & TA_ANT & $\mathbf{2 5 ; 7 5 \%}$ & TA_D & $\mathbf{2 5 ; 7 5 \%}$ & TA_E & $\mathbf{2 5 ; 7 5 \%}$ \\
\hline G1 & 54,5 & $(54 ; 55)$ & 22 & $(17 ; 25)$ & 17,5 & $(14 ; 19)$ & 16 & $(14 ; 18)$ \\
G2 & 55,5 & $(55 ; 56)$ & 25,5 & $(22 ; 27)$ & 18,5 & $(17 ; 20)$ & 18 & $(16 ; 21)$ \\
G3 & 56 & $(55 ; 56)$ & 24 & $(21 ; 31)$ & 21,5 & $(18 ; 23)$ & 20 & $(17 ; 23)$ \\
G4 & 52 & $(45 ; 54)$ & 19 & $(12 ; 25)$ & 15 & $(11 ; 19)$ & 13,5 & $(9 ; 19)$ \\
\hline P & 0,000 & & 0,000 & & 0,001 & & 0,001 & \\
\hline
\end{tabular}


Figura 1. Resultados dos testes BOA sobre a PF, variável COP $\left(\mathrm{cm}^{2}\right)$, em medianas e percentis (25\% e $75 \%) \mathrm{e}^{*}$ indica as diferenças significantes quando comparados individualmente, valor de p Kruskal-Wallis.

\section{BOA COP}

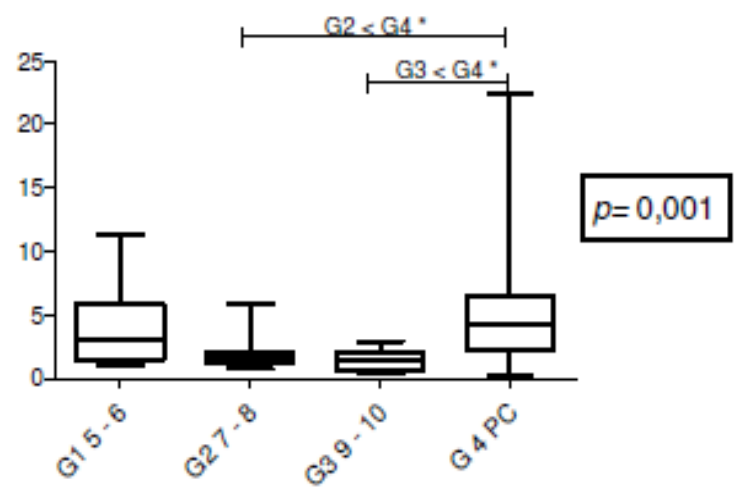

\section{DISCUSSÃO}

Observou-se que a PC interfere negativamente no controle postural, quando avaliado por meios funcionais e parâmetros estabilográficos. Na EEP, o fator que influenciou as respostas do G4 foi o grau de comprometimento motor. A variação da pontuação do G4 pode estar relacionada ao fato da constituição do grupo ser de crianças com idade entre cinco a 12 anos e comprometimento motor leve e moderado, ou seja, em algumas tarefas a dificuldade para a execução foi maior nos participantes com a condição motora mais afetada. Quanto maior o comprometimento, conforme a avaliação pelo GMFCS, maior será a limitação funcional nas crianças com $\mathrm{PC}^{15}$.

Em relação ao TA, as crianças com PC tem menor amplitude de movimento de ombro, que é contrabalanceado com movimentos compensatórios de tronco, para obter maior alcance ${ }^{16}$. Tal compensação, provavelmente, desafia ainda mais o equilíbrio, pelo fato do centro de gravidade ser retirado da base de suporte. Outros fatores de interferência nas respostas do TA das crianças com PC podem ser a restrição da amplitude de movimento, devido a presença de retraçóes e contraturas e espasticidade $^{17}$.

Sobre a PF na posição BOA, o G4 mostrou maior dificuldade de controle postural, mesmo sendo a postura simples e estável, sendo ainda o G4 mais velho que G1. Tais resultados também foram encontrados em testes
Figura 2. Resultados dos testes UNID sobre a PF, variável COP $\left(\mathrm{cm}^{2}\right)$, em medianas e percentis $(25 \%$ e $75 \%)$ e ${ }^{*}$ indica as diferenças significantes quando comparados individualmente, valor de p Kruskal-Wallis.

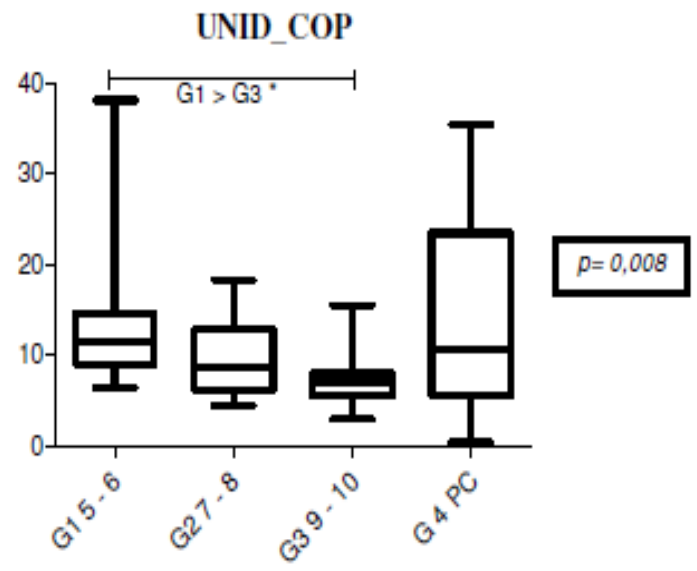

funcionais que demostraram que a criança com PC, com idade de 6,5 anos é funcionalmente semelhante a criança recém deambuladora de 1,1 ano $^{18}$. No teste UNID o G4 também mostrou pior desempenho e grande variação, principalmente, em relação a G2 e G3, provavelmente a grande variação foi influenciada pelo grau de comprometimento, sendo que crianças com PC leve, possivelmente conseguem melhor resposta, já o moderado tem maiores dificuldades no controle postural ${ }^{15}$. Já no UNIE a área do COP e VEL_AP_ML de G4 foi ligeiramente menor do que dos grupos G1, G2 e G3, teoricamente, os resultados deveriam ser piores, porém na prática foi observado que

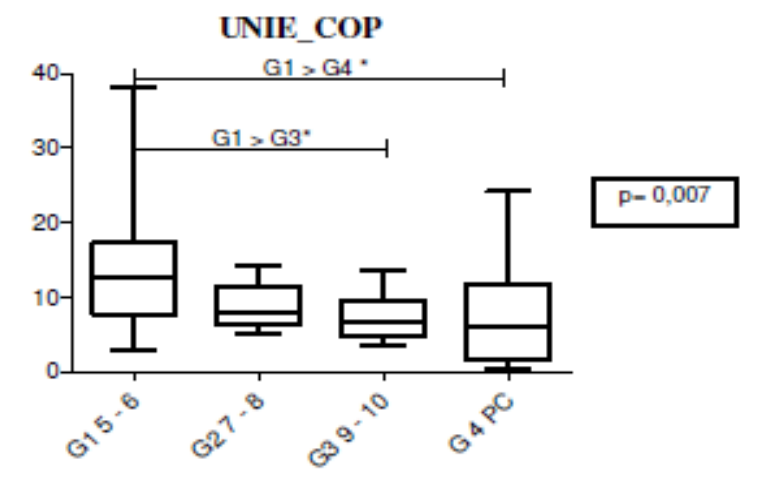

Figura 3. Resultados dos testes UNIE sobre a PF, variável COP $\left(\mathrm{cm}^{2}\right)$, em medianas e percentis $(25 \%$ e $75 \%)$ e $^{*}$ indica as diferenças significantes quando comparados individualmente, valor de $\mathrm{p}$ Kruskal-Wallis. 
as crianças do G4 que apresentavam hemiplegia à esquerda $(n=5)$ tinham melhor controle postural sobre o membro afetado, talvez por existir uma ênfase de treinamento de transferência de peso sobre o lado comprometido ${ }^{19}$. Ainda, as crianças com PC geralmente iniciam a fisioterapia precocemente, sendo muito mais estimulada a ganhar controle postural, possivelmente, para uma criança mais nova com DT seria novidade ${ }^{20}$. A falta de controle seletivo do membro inferior afetado quando em apoio contralateral, ou seja, UNID pode interferir nos resultados, autores mostram que a falta de sinergismo e coordenação desafiam o centro de gravidade, promovendo uma instabilidade ainda maior nessas crianças ${ }^{21}$. Também, em um estudo de análise do desempenho motor de crianças com PC, foi identificado que o ortostatismo e a marcha podem apresentar sobrecarga do lado não afetado, por suportar maior parte do peso corporal ${ }^{22}$.

Os resultados desse estudo podem ser explicados pelo fato que, a PC altera o repertório motor, diminuindo e dificultando o número de estratégias e habilidades que, possivelmente, estão relacionadas à performance do controle postural ${ }^{23}$. Em relação ao processo de maturação, à medida que avança a idade ocorre a melhora do desempenho de controle postural nas crianças em desenvolvimento típico, tanto em testes funcionais, quanto nos parâmetros estabilográficos.

$\mathrm{Na}$ EEP e no TA as crianças G1, em idade pré-escolar, pontuaram menos que as em idade escolar. Analises anteriores identificam a melhora no desempenho do teste funcional do alcance anterior ${ }^{8,12}$. Outro ponto importante foi que, dentre as crianças em DT, G1 obteve maior variação, em que as crianças mais novas se comportaram semelhantemente as do nosso estudo ${ }^{24}$.

Sobre a PF, tanto em postura simples, como a bipodal, quanto na mais instável, unipodal, o G1, na maioria dos parâmetros, obteve pior resultado, mostrando que o controle postural de uma criança entre cinco e seis anos se encontra em processo de maturação ${ }^{7,25}$. Ambos ressaltam que a maturação dos sistemas estão diretamente ligados a idade, assim, o melhor controle postural ocorre quando os sistemas estão mais aprimorados.

Uma das limitaçóes do estudo foi que nas posiçôes UNIE e UNID para o G4, três crianças com diplegia espástica de grau moderado, só conseguiram realizar os testes com apoio de membros superiores, assim, foram excluídos, diminuindo o número de participantes. Outra limitação foi o tamanho da amostra do G4, em função dos critérios de inclusão e exclusão, não permitindo a separação em dois grupos e algumas comparaçóes, como por exemplo, grupo de crianças com hemiplegia e diplegia, hemiplegia a esquerda com a direita e diferentes graus de comprometimento, conforme o GMFCS.

\section{CONCLUSÃO}

Conclui-se que a PC afeta o controle postural de crianças, e que na prática clinica deve ser dado ênfase no desenvolvimento do controle postural, seja utilizando métodos convencionais, seja por meio de outras abordagens. Outro aspecto do tratamento refere-se a melhor manutenção na posição unipodal em crianças com PC, visto ser necessário o treinamento de transferência de peso e também de controle seletivo do membro afetado, quando em apoio contralateral.

\section{REFERÊNCIAS}

1.Manoel EJ. Motor development: changing patterns, increasing complexity. Rev Paul Educ Fis 2000;3:35-54.

2.Fetters L. Perspective on variability in the development of human action. Phys Ther 2010;90:1860-7. http://dx.doi.org/10.2522/ptj.2010090

3.Hiratuka E, Matsukura TS, Pfeifer LI. Cross-cultural adaptation of the Gross Motor Function Classification System into Brazilian-Portuguese (GMFCS). Rev Bras Fisioter 2010;14:537-44. http://dx.doi.org/10.1590/S1413$\underline{35552010000600013}$

4.Assis-Madeira EA, Carvalho SG. Cerebral palsy and risks factors in motor development: A theoretical review. J Post Dev Disord 2009;9:142-63. http:// dx.doi.org/10.1016/j.ridd.2009.01.004

5.Schwarztman JS. Cerebral palsy. Bras Arch Cer Palsy 2004;1:4-17.

6.Woollacott M, Shumway-Cook A, Hutchinson S, Ciol M, Price R, Kartin D. Effect of balance training on muscle activity used in recovery of stability in children with cerebral palsy: a pilot study. Dev Med Child Neurol 2005;47:455-61. http://dx.doi.org/10.1111/j.1469-8749.2005.tb01171.x

7.Steindl RMD, Kunz KMD, Schrott-Fischer A, Scholtz AW. Effect of age and sex on maturation of sensory systems and balance control. Dev Med Child Neurol 2006;48:477-82. http://dx.doi.org/10.1111/j.1469-8749.2006. tb01299.x

8.Franjoine MR, Gunther JS, Taylor MJ. Pediatric Balance Scale: A Modified Version of the Berg Balance Scale for the School-Age Child with Mild to Moderate Motor Impairment. Ped Phys Ther 2003;15:114-28.http://dx.doi. org/10.1097/01.PEP.0000068117.48023.18

9.Mancine M, Horak FB. The relevance of clinical balance assessment tools to differentiate balance deficits. Eur J Rehabil Med 2010;46:239-48.

10.Ries LGK, Michaelsen SM, Soares PSA, Monteiro VC, Allegretti KMG. Cross-cultural adaptation and reliability analysis of the Brazilian version of 
Pediatric Balance Scale (PBS). Rev Bras Fisioter 2012;16:14. http://dx.doi. org/10.1590/S1413-35552012005000026

11.Duncan PW, Weiner DK, Chandler J, Studenski S. Functional reach: a new clinical measure of balance. J Gerontol 1990;45:192-7. http://dx.doi. org/10.1093/geronj/45.6.M192

12.Bartlett D, Birmingham T. Validity and Reliability of a Pediatric Reach Test. Pediatr Phys Ther 2003;15:84-92. http://dx.doi.org/10.1097/01. PEP.0000067885.63909.5C

13.da Silva RA, Bilodeau M, Parreira RB, Teixeira DC, Amorin CF. Age-related differences in time-limit performance and force platform-based balance measure during one-leg stance. J Electromyogr Kinesiol 2013;23:634-9. http://dx.doi.org/10.1016/j.jelekin.2013.01.008

14.Bauer C, Groger I, Rupprecht R, Gassman KG. Intrasession reliability of force platform parameters in community-dwelling older adults. Arch Phys Med Rehabil 2008;89:1977-82. http://dx.doi.org/10.1016/j.apmr.2008.02.033

15.Majnemer A, Shevell M, Hall N, Poulin C, Law M. Developmental and functional abilities in children with cerebral palsy as related to pattern and level of motor function. J Child Neurol 2010;25:1236-41. http://dx.doi. $\underline{\text { org } / 10.1177 / 0883073810363175}$

16.Coluccini M, Maini ES, Martelloni C, Sgandurra G, Cioni G. Kinematic characterization of functional reach to grasp in normal and in motor disabled children. Gait Posture 2007;25:493-501. http://dx.doi.org/10.1016/j.gaitpost.2006.12.015

17.Zaino CA, McCoy SW. Reliability and comparison of electromyographic and kinetic measurements during a standing reach task in children with and without cerebral palsy. Gait Posture 2008;27:128-37. http://dx.doi. org/10.1016/j.gaitpost.2007.03.003
18.Burtner PA, Woollacott MH, Craft GL, Roncsvalles NM. The capacity to adapt to changing balance threats: A comparison of children with cerebral palsy and typically developing children. Dev Neurorehabil 2007;10:249-60. http://dx.doi.org/10.1080/17518420701303066

19.Papavasiliou AS. Management of motor problems in cerebral palsy: A critical update for the clinician. Eur J Pediatr Neuro 2009;13:387-96. http:// dx.doi.org/10.1016/j.ejpn.2008.07.009

20.Nakaya L, Mazzitelli C, Sá CSC. Comparação do Equilíbrio de Crianças com Paralisia Cerebral e Crianças com Desenvolvimento Motor Normal. Rev Neurocienc 2013;21:510-9. http://dx.doi.org/10.4181/RNC.2013.21.822.10p

21.Ferdjallah M, Harris GR, Smith P,Wertsch JJ. Analysis of postural control synergies during quiet standing in healthy children and children with cerebral palsy. Clin Biomech 2002;17:203-10. http://dx.doi.org/10.1016/S0268$\underline{0033(01) 00121-8}$

22.Silva FPP, Gomes CNM, Gil KVC, Da Matta DSP. Comparação do Desempenho Funcional de Crianças com Paralisia Cerebral Diparéticas e Hemiparéticas. Rev Neurocienc 2012;20:511-6. http://dx.doi.org/10.4181/ RNC.2012.20.725.6p

23.Bruggink JL, Cioni G, Einspieler C, Maathuis CG, Pascale R, Bos AF. Early motor repertoire is related to level of self-mobility in children with cerebral palsy at school age. Dev Med Child Neurol 2009;51:878-85. http://dx.doi. org/10.1111/j.1469-8749.2009.03294.x

24.Franjoine MR, Darr N, Held SL, Kott K, Young BL. The performance of children developing tipically on the pediatric balance scale. Pediatr Phys Ther 2010;22:350-9. http://dx.doi.org/10.1097/PEP.0b013e3181f9d5eb.

25.Mickle KJ, Munro BJ, Steele JR. Gender and age affect balance performance in primary school-aged children. J Sci Med Sport 2011;14:243-8. http:// dx.doi.org/10.1016/j.jsams.2010.11.002 